Féeries

Études sur le conte merveilleux, XVII -XIXe siècle

$12 \mid 2015$

À la croisée des genres

\title{
Le roman et les sortilèges : réemplois du conte merveilleux chez George Sand et Jules Barbey d'Aurevilly
}

The Novel and the Spells: Re-uses of the Supernatural Tale in the Works of George Sand and Jules Barbey d'Aurevilly

Julie Anselmini

\section{OpenEdition}

Journals

Édition électronique

URL : http://journals.openedition.org/feeries/965

ISSN : 1957-7753

Éditeur

UGA Éditions/Université Grenoble Alpes

Édition imprimée

Date de publication : 15 octobre 2015

Pagination : $57-77$

ISBN : 978-2-84310-306-3

ISSN : 1766-2842

Référence électronique

Julie Anselmini, « Le roman et les sortilèges : réemplois du conte merveilleux chez George Sand et Jules Barbey d'Aurevilly », Féeries [En ligne], 12 | 2015, mis en ligne le 15 octobre 2016, consulté le 08 septembre 2020. URL : http://journals.openedition.org/feeries/965 


\section{LE ROMAN ET LES SORTILÈGES : RÉEMPLOIS DU CONTE MERVEILLEUX CHEZ GEORGE SAND ET JULES BARBEY D'AUREVILLY}

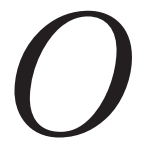

N NE PEUT GUĖRE SOUPÇONNER BARBEY D'AUREVILLY — connu du reste pour ses vitupérations pamphlétaires — de sympathie à l'égard de Sand; dans ses Bas-bleus, il fait d'elle une représentante particulièrement odieuse de cette catégorie monstrueuse de femmes dévoyées ${ }^{\mathrm{I}}$. Pourtant, des parentés évidentes rapprochent les deux écrivains : l'ancrage de certaines de leurs œuvres dans un terroir lié à leurs origines familiales et à leur enfance (le Berry pour Sand, la Normandie pour Barbey), leur intérêt pour le patois local ou encore, et c'est ce dernier point qui nous intéressera au premier chef, le fait qu'ils sont des romanciers-conteurs. Non seulement, en effet, ces deux auteurs cultivèrent en tant que tel le genre du conte, auquel on peut assimiler les nouvelles des Diaboliques de Barbey (1874) et dont relèvent à part entière les Contes d'une grand-mère dédiés par Sand à ses petites-filles (I873 et I876), mais il est encore évident que leur pratique romanesque est nourrie par l'influence de ce modèle générique. Tous deux revendiquent d'ailleurs, en tant que romanciers ou pour certains de leurs romans, le statut de conteurs ou de contes. La Mare au Diable, François le Champi et La Petite Fadette sont ainsi présentés, dans la préface de l'édition originale de cette dernière ouvre, comme "une série de contes villageois, [classiquement intitulés] Les Veillées du Chanvreur $^{2}$; et dans la notice composée pour la publication du roman

I. Voir «Mme George Sand jugée par elle-même», dans Les Euvres et les Hommes. se partie: Les Bas-bleus, Paris, Victor Palmé, I878, p. 45-6I. Sur les relations entre les deux auteurs, voir M. Bertrand, "Jules et George : les dessous d'une animosité", Littératures, n ${ }^{\circ} 58-59$ (Barbey polémiste, P. Glaudes et M.-C. Huet-Brichard [dir.]), 2008, p. 37-5I.

2. Préface de l'édition originale, La Petite Fadette, A. Mansau (éd.), dans B. Didier (dir.), G. Sand, Euvres complètes. I849, Paris, Honoré Champion, coll. "Textes de littérature moderne et contemporaine», 20I3, p. 2I4. Toutes les références à La Petite Fadette renvoient à cette édition. 
dans Les Euvres illustrées de Sand (Hetzel, I852), il est encore question "du conte qu'on va lire" $»$. Quant au narrateur de L'Ensorcelée, il affirme au chapitre IX : "Je ne suis qu'un simple conteur ${ }^{4}$.»

En nous fondant sur deux romans publiés à peu d'années de distance, La Petite Fadettes de Sand et L'Ensorcelée $e^{6}$ de Barbey d'Aurevilly, romans dont nous verrons qu'ils ne sont pas sans points communs par leurs intrigues et par les motifs mis en œuvre 7 , nous verrons comment une semblable influence du conte et des similitudes notables dans l'intégration du merveilleux à la trame des récits aboutissent néanmoins à des esthétiques et des visions du monde radicalement distinctes.

\section{Charmes et sortilèges}

L'influence thématique du conte merveilleux est tout d'abord patente chez Barbey comme chez Sand, où l'on trouve déclinée toute une série de figures et de motifs magiques.

Dans L'Ensorcelée, le titre met en exergue le thème traditionnel de l'ensorcellement : celui de Jeanne Le Hardouey, qui tombe si passionnément amoureuse de Jéhoël de la Croix-Jugan, ancien Chouan monstrueusement défiguré par une tentative de suicide et par les tortures infligées par les Bleus, qu'elle jette non seulement par-dessus les moulins sa coiffe d'épouse honorable et de bonne maîtresse de maison, mais qu'une fois éconduite par cet inflexible abbé, elle finit noyée, sans doute suicidée, au fond d'un étang.

Trois figures de sorciers se voient attribuer dans le roman la responsabilité de ce sortilège. La première d'entre elles est Clotilde Mauduit, ancienne fille perdue, devenue infirme, qui vit dans un taudis isolé, devant un antique chaudron où bout sa pitance, et auprès d'un rouet qui l'appa-

3. Ibid., "Notice», p. 48.

4. J. Barbey d'Aurevilly, Euvres romanesques complètes, J. Petit (éd.), Paris, Gallimard, coll. «Bibliothèque de la Pléiade», I964, t. I, p. 658. Toutes les références à L’Ensorcelée renvoient à cette édition.

5. G. Sand, La Petite Fadette, Paris, Michel Lévy frères, I849; pré-originale dans Le Crédit, I848-I849.

6. J. Barbey d'Aurevilly, L'Ensorcelée, Paris, Cadot, I855; pré-originale dans L'Assemblée nationale, I852.

7. Le cadre de cette étude ne permet pas d'étendre celle-ci à d'autres œuvres où l'on pourrait examiner les réemplois du conte merveilleux, notamment La Mare au Diable de Sand (1846) et Une Vieille maîtresse (185I), ou Un Prêtre marié (1865) de Barbey d'Aurevilly. 
rente à l'une de ces Parques filant la destinée des hommes ${ }^{8}$; paralysée à partir de la taille, et se traînant pour se mouvoir à l'aide d'un bâton, elle semble aussi, de par sa description physique, en cours de métamorphose, pétrifiée à un stade hybride de sa transformation :

À côté de son fauteuil, on voyait son bâton d'épine durcie au four sur lequel elle appuyait ses deux mains, quand, avec des mouvements de serpent à moitié coupé qui tire son tronçon en saignant, elle se traînait jusqu'au feu de tourbe de sa cheminée?.

Son patronyme connote en outre la malédiction, qu'elle passe pour avoir elle-même exercée à l'encontre de Jeanne; venue assister à l'enterrement de celle-ci, elle est interpellée en ces termes par un boucher : "Vieille sorcière! [...] est-ce pour maléficier aussi son cadavre que tu t'en viens $[\ldots]$ à l'enterrement d'une femme que tu as ensorcelée $[. . .]^{10}$ ? ${ }^{\text {" C'est enfin }}$ en sorcière qu'elle finit lapidée, aux cris de : «À mort, la vieille sorcière ${ }^{\mathrm{II}}$ !", mise en pièces après avoir été attachée sur une claie traînée par des hommes lancés au galop.

Le narrateur, pourtant (sur la position de qui nous reviendrons), n'impute à Clotilde Mauduit aucun maléfice contre Jeanne, qu'elle traite au contraire avec une tendresse maternelle. "Maîtresse Le Hardouey", en revanche, est l'objet de la haine rancunière d'un de ces pâtres errants, d'origine immémoriale, à qui les paysans du Cotentin prêtent des pouvoirs diaboliques :

Il y a dans la presqu'île du Cotentin (depuis combien de temps? on l'ignore?) de ces bergers errants qui se taisent sur leur origine et qui se louent pour un mois ou deux dans les fermes [...]. Espèces de pâtres bohémiens, auxquels la voix du peuple des campagnes attribue des pouvoirs occultes et la connaissance des secrets et des sortilèges. [...] Si par hasard un fermier les expulse durement de son service ou ne veut plus les employer, ils ne disent mot, courbent la tête et s'éloignent; mais un doigt levé, en se retournant, est leur seule et sombre menace; et presque toujours un malheur, soit une mortalité parmi les bestiaux, soit les fleurs de tout un plant de pommiers brûlées dans une nuit, soit la corruption de l'eau des fontaines, vient bientôt suivre la menace du terrible et silencieux doigt levér ${ }^{12}$.

8. Voir sur ce point P. Jonchière, «Splendeurs et misères de la magicienne chez Barbey d'Aurevilly", dans S. Bernard-Griffiths et J. Guichardet (dir.), Images de la magie. Fées, enchanteurs et merveilleux dans l'imaginaire du XIX ${ }^{e}$ siècle, Annales littéraires de l'université de Besançon, $\mathrm{n}^{\circ}$ 504, Paris, Les Belles Lettres, 1993, p. 90.

9. J. Barbey d'Aurevilly, L'Ensorcelée, éd. citée, chap. vII, p. 635.

Io. Ibid., chap. XIII, p. 705 .

II. Ibid., chap. XIII, p. 707.

I2. Ibid., chap. II, p. 575-576. 
À ce pâtre est attribuée au cours du récit une série de sortilèges, visant à assouvir sa vengeance vis-à-vis de Jeanne et surtout du mari de celle-ci, riche propriétaire qui a repoussé avec mépris ses offres de service. C’est d'abord la funeste prédiction qui est faite à Jeanne, au retour des vêpres de Blanchelande où elle rencontre pour la première fois l'abbé de la CroixJugan, prédiction qui a toutes les allures d'une malédiction :

Il y a de meilleures vengeances, et plus sûres. La corne met du temps à venir au tauret, et ses coups n'en sont que plus mortels. Allez! marchez! — insista-t-il d'une voix sinistre. - Vous vous souviendrez longtemps des vêpres d'où vous sortez, maîtresse Le Hardouey ${ }^{13}$ !

Puis, au chapitre XI, alors que Jeanne, envoûtée par l'abbé, n'est déjà plus que l'ombre d'elle-même, le berger, entouré de ses compagnons, opère un "charme» devant maître Le Hardouey; dans son miroir magique, et après avoir prononcé tout bas "des mots étranges ${ }^{14}$ » il montre au mari de Jeanne son propre cœur, mis à la broche et piqué au couteau par la jeune femme et par l'abbé, "vision [...] si horrible que Le Hardouey se sentit frappé d'un coup de massue à la tête, et [...] tomba à terre comme un bœuf assommé ${ }^{15} »$. Une autre manifestation du pouvoir du pâtre est sa prémonition, devant l'étang des lavandières, que le corps de Jeanne y repose, prémonition bientôt vérifiée. Certes, le vagabond a pu épier le suicide de la jeune femme; néanmoins, la joie féroce qu'il laisse éclater devant son cadavre laisse penser qu'il l'a bien découvert en même temps que les lavandières, et qu'un sixième sens magique l'a averti de sa présence au fond de l'eau ${ }^{16}$. Enfin, au chapitre XIv, Thomas Le Hardouey renie son incrédulité ${ }^{17}$ et obtient du pâtre la prédiction selon laquelle l'abbé de la Croix-Jugan périra d'une balle ${ }^{\mathrm{r}}$, ce qui ne manque pas de se réaliser. Il est vrai que le berger pouvait se douter que maître Le Hardouey chercherait à tuer l'abbé, et qu'il apparaît expert dans l'art de la manipulation et de la tromperie : la recette magique qu'il a donnée à Jeanne pour se faire aimer de Jéhoël (lui faire porter une chemise macérée dans la sueur de la jeune

13. Ibid., chap. vI, p. 620.

I4. Ibid., chap. XI, p. 678.

15. Ibid., chap. XI, p. 679.

I6. Ibid., chap. XII, p. 690-692.

17. "J'ai cru longtemps qu'il n'y avait pas d'âme, qu'il n'y avait pas de Satan non plus", dit-il au berger. «Mais ce que les prêtres n'avaient jamais su faire, tu l'as fait, toi! Je crois au démon, et je crois à vos sortilèges, canailles de l'enfer! [...] Vous avez du pouvoir. Je l'ai éprouvé...» (Ibid., p. 719.)

18. Ibid., chap. XIV, p. 72 I. 
femme) s'est avérée inefficace ${ }^{19}$, faisant de Jeanne une «Médée inopérante et privée de pouvoir».

Une dernière figure de sorcier est l'abbé de la Croix-Jugan lui-même, dont la vie "prêtait merveilleusement aux imaginations étranges ${ }^{20}$ ", et à qui la population impute l'ensorcellement de Jeanne : «Il n'y a pas moyen de penser que le maître de cette misérable ensorcelée ait été un autre que ce prêtre, qui semblait le démon en habit de prêtre», juge maître Tainnebouy ${ }^{21}$. Le premier phénomène surnaturel mentionné dans le récit, et enclenchant celui-ci, lui est en outre relié puisqu'il s'agit de la sonnerie des cloches retentissant mystérieusement dans la lande de Lessay sitôt frappé minuit ${ }^{22}$, pour célébrer "une messe des Morts, sans répons et sans assistance, une terrible et horrible messe», «la messe nocturne de l'abbé de la Croix-Jugan ${ }^{23}$ ».

Dans La Petite Fadette de Sand - qui rappelle dans Histoire de ma vie quel plaisir elle éprouvait, enfant, à la lecture des contes de Madame d'Aulnoy et de Perrault ${ }^{24}$ —, on trouve déjà deux figures de sorcières. D'une part, la mère Fadet, "cette vieille charmeuse, [...] toujours en malice ${ }^{25}$ ", "qui demeurait tout au bout de la Joncière» et "pansait $d u$ secret ${ }^{26}$ ", c'està-dire savait soigner diverses blessures par des paroles magiques. D'autre part, sa petite-fille, l'héroïne éponyme du roman, ainsi nommée par les paysans, "grands donneurs de sornettes [surnoms] et sobriquets ${ }^{27}$ » : «[...] on [l'] appelait dans le pays la petite Fadette, autant pour ce que c'était son nom de famille que pour ce qu'on voulait qu'elle fût un peu sorcière aussi ${ }^{28}$.» Mais la petite fille est également rapprochée d'autres créatures merveilleuses, comme le révèle la suite du passage :

Vous savez tous que le fadet ou le farfadet [...] est un lutin fort gentil, mais un peu malicieux. On appelle aussi fades les fées [...]. Mais que cela voulût dire une petite

19. Voir chap. X, p. 668-669. De même, les "poudres" que les «faiseuses de breuvage" donnèrent jadis à Dlaïde Malgy pour se faire aimer de Jéhoël «étaient des menteries» (ibid., chap. VII, p. 64I).

20. J. Barbey d'Aurevilly, L'Ensorcelée, éd. citée, chap. IX, p. 652.

2I. Ibid., chap. IX, p. 663.

22. Ibid., chap. II, p. 58I-582.

23. Ibid., chap. II, p. 582. Lorsque le roman parut en feuilleton dans le journal L'Assemblée nationale, le titre en était alors La Messe de l'abbé de la Croix-Jugan.

24. Histoire de ma vie, dans Euvres autobiographiques, G. Lubin (éd.), Paris, Gallimard, coll. «Bibliothèque de la Pléiade», I970, t. I, p. 618.

25. G. Sand, La Petite Fadette, éd. citée, chap. C, p. 95.

26. Ibid., chap. vIII, p. 83 .

27. Ibid., chap. IV, p. 67.

28. Ibid., chap. VIII, p. 84-85. 
fée, ou la femelle du lutin, chacun en la voyant s'imaginait voir le follet, tant elle était petite, maigre, ébouriffée et hardie ${ }^{29}$.

Les figures sollicitées par Sand sont ainsi plus variées et relèvent d'un merveilleux plus riant et fantaisiste. Quant aux pratiques magiques prêtées à la petite Fadette, soupçonnée d'accointances avec les esprits malins, il s'agit essentiellement de l'ensorcellement de Landry Barbeau, un des jumeaux de la Bessonnière, qui passe pour le plus beau gars du pays. Ces rumeurs naissent dès la Saint-Andoche, alors que Landry fait danser la jeune fille, grotesquement attifée : "Et parlant à Landry, il y en eut qui dirent : - Elle t'a donc jeté un sort, mon pauvre Landry, que tu ne regardes qu'elle? ou bien c'est que tu veux passer sorcier, et que bientôt nous te verrons mener les loups aux champs ${ }^{30}$." Après sa première longue entrevue en tête-à-tête avec Fanchon, qu'il a embrassée, Landry lui-même "était comme affolé», et «sentant que le diable était après lui, il se mit à courir ${ }^{31}$; ; le lendemain, au souvenir de ses émotions violentes de la veille, il se tient à part lui le monologue suivant : "Il faut qu'elle soit charmeuse, comme on le dit, [...] car pour sûr elle m’a ensorcelé hier soir, et jamais $[\ldots]$ je n'ai senti $[. .$.$] un élan d'amitié pareil à celui que [...] cette dia-$ blesse m’a causéz ${ }^{2}$.» Quant au narrateur du récit, il juge lui aussi Landry "ensorcelé33".

Chez Sand comme chez Barbey, la passion amoureuse (qui dans les deux cas est au cour du roman) fait ainsi l'objet d'une lecture magique. Ses effets, cependant, bien différents, révèlent des divergences notables dans le statut des figures magiques et du merveilleux. Chez Barbey, la magie n'a de conséquences que néfastes; c'est une magie noire qui conduit infailliblement à la mort des protagonistes : Jeanne, mais aussi deux de ses supposés ensorceleurs, Clotilde Mauduit et Jéhoël de la Croix-Jugan. Chez Sand, au contraire, la Fadette apporte en définitive à Landry la sagesse, la vertu, le bonheur et même la richesse; la fin de La Petite Fadette, à l'exception du sort plus ambigu réservé à Sylvinet Barbeau ${ }^{34}$, est celle d'un conte de fées

29. Ibid., chap. VIII, p. 85 .

30. Ibid., chap. Xvi, p. I20.

31. Ibid., chap. xx, p. I39.

32. Ibid., chap. xx, p. I39. La revoyant quelques jours après à la messe, transfigurée, il se fait à nouveau cette réflexion : «Elle est sorcière; elle a voulu devenir belle de laide qu'elle était, et la voilà belle par miracle.» (Ibid., chap. XxII, p. I46)

33. Ibid., chap. xxv, p. 156.

34. Voir sur ce point P. Laforgue, «Le roman infini. Narratif, romanesque et réflexivité métapoétique chez Sand (Quelques exemples et quelques propositions) », dans B. Diaz et I. Hoog-Naginski 
classique : ayant hérité d'un trésor à la mort de sa grand-mère, Fanchon épouse Landry et ils vivent heureux, entourés de beaux enfants. Avant ce dénouement nuptial, qui lui permet de prouver la mesure de sa charitée $^{35}$, Fanchon, vertueuse et pieuse ${ }^{36}$, a révélé des dons thaumaturgiques en soignant Sylvinet, dévoré par la fièvre, par une simple imposition des mains ${ }^{37}$, se révélant "une remégeuse plus habile que toutes celles du pays, et qui ne voulait point exercer pour de l'argent [...], mais pour le seul amour du bon Dieu et du prochain ${ }^{38} »$ : c'est une magie blanche que la jeune fille pratique et qui lui permet de "charmer la maladie ${ }^{39}$ ».

\section{Vraisemblance du merveilleux}

Si des orientations opposées se dessinent dans le traitement du merveilleux (maléfique et mortifère chez Barbey, bénéfique et régénérateur chez Sand), les deux romanciers sont confrontés à un problème identique. Dans le cadre du conte, le surnaturel s'intègre sans heurt à un univers qui lui est homogène; cette cohérence interne fait du merveilleux du conte, selon les analyses désormais canoniques de Tzvetan Todorov ${ }^{40}$, un merveilleux accepté sans aucun mal par le lecteur. En revanche, dans le cadre du roman, le surnaturel doit s'intégrer à un univers réaliste, univers suscitant chez le lecteur une illusion référentielle fondée sur la vraisemblance et différente, en cela, de la crédulité ludique que met en œuvre le conte. Ainsi, l'action de L'Ensorcelée s'insère dans un cadre historique authentifié : le chapitre III du roman s'ouvre en l'an VI de la République, dans le contexte d'une «arrière-chouannerie» qui voit s'éteindre ses derniers feux et dont Jéhoël de la Croix-Jugan est l'un des derniers combattants; l'action principale commence quelque temps plus tard, "[a]près la guerre de la Chouannerie, et lorsqu'on rouvrit les églises ${ }^{41}$ ». De même, "malgré leurs mentions implicites, les tragédies de l'Histoire, guerres et révolutions ne sont pas absentes de La Petite Fadette ${ }^{42} »$, et le roman de Sand comporte maints éléments

(dir.), George Sand. Pratiques et imaginaires de l'écriture, Caen, Presses universitaires de Caen, 2006, p. 337-338.

35. Voir La Petite Fadette, éd. citée, chap. XL, p. 208.

36. Voir ibid., chap. xv, p. II7, où ses longues prières et sa dévotion sont mentionnées.

37. Voir ibid., chap. XxxIV, p. I9I-I92 et XXXv, p. I93-I94.

38. Ibid., chap. xxxiv, p. I9I.

39. Ibid., chap. xxxix, p. 205.

40. Voir son Introduction à la littérature fantastique, Paris, Éditions du Seuil, coll. «Points», I970.

4I. J. Barbey d'Aurevilly, L'Ensorcelée, éd. citée, chap. III, p. 598.

42. A. Mansau, «Présentation» de La Petite Fadette, éd. citée, p. 24. 
réalistes de contextualisation : «Nous avons eu, dans nos pays, une suite de mauvaises années, tant pour les vimaires du temps [intempéries] que pour les embarras du commerce, qui ont délogé plus d'écus de la poche des gens de campagne qu'elles n'y en ont fait rentrer", lit-on par exemple au chapitre $\mathrm{II}^{43}$. Dès lors, le romancier doit inventer des ruses pour réduire l'hétérogénéité des deux composantes et introduire de manière convaincante le surnaturel sans violer la cohérence du roman. Parmi ces ruses, l'une consiste à ménager des zones de transition entre l'univers réaliste et les phénomènes merveilleux, comme le suggère Hubert Matthey ${ }^{44}$.

C'est le rôle, dans les deux œuvres qui nous occupent, du décor planté par les romanciers. L'action se déroule en effet dans une région éloignée de Paris : le Berry pour Sand et le Cotentin pour Barbey. Dans la longue description liminaire de L'Ensorcelée, le narrateur souligne la sauvagerie de la lande de Lessay et remarque d'emblée à propos de "ces terres chauves»: «On ne saurait dire l'effet qu' elles produisent sur l'imagination de ceux qui les traversent, de quel charme bizarre et profond elles saisissent les yeux et le cœur. Qui ne sait le charme des landes?... ${ }^{45}$ ", répétant significativement le terme polysémique "charme». «[...] la lande, terrain des mystères, la possession des esprits, la lande incessamment arpentée par les pâtres rôdeurs et sorciers ${ }^{46}{ }^{»}$ est ainsi le premier acteur du roman. Ce lieu coupé des zones habitées, "seuil spatial entre deux mondes ${ }^{47}$ ", est rendu plus fantasmagorique encore par la tombée de la nuit, lorsque le narrateur y entend pour la première fois de maittre Tainnebouy l'histoire de Jeanne : «Il y avait à peu près une heure que nous chevauchions dans la lande, et le brouillard avait fini par nous envelopper complètement de son réseau diaphane. La lune filtrait dans la vapeur une lumière pâle et incertaine ${ }^{48}$.» L'influence de cette atmosphère fantomatique est du reste soulignée par le narrateur :

43. Ibid., chap. II, p. 59-60.

44. "L'auteur qui, de but en blanc, sans préambule et sans précaution, introduirait au milieu d'un récit réaliste des êtres ou des événements surnaturels, loin de nous convaincre de leur vérité, ne ferait croire qu'à sa folie.» Essai sur le merveilleux dans la littérature française depuis I80o. (Contribution à l'étude des genres), Lausanne, Librairie Payot et $\mathrm{C}^{\mathrm{ie}}$, 1915 , p. 279.

45. J. Barbey d'Aurevilly, L'Ensorcelée, éd. citée, chap. I, p. 555.

46. Ibid., chap. XIII, p. 709.

47. C. Bricault, «Un chronotope de la rencontre magique : le seuil dans les romans normands de Barbey d'Aurevilly", dans S. Bernard-Griffiths et C. Bricault (dir.), Magie et magies dans la littérature et les arts du XIXe siècle français, ouvr. cité, p. I9I.

48. J. Barbey d'Aurevilly, L'Ensorcelée, éd. citée, chap. II, p. 57I. 
Était-ce l'heure à laquelle un croyant à cette épouvantable vision [de la messe-fantôme de l'abbé] me la racontait? Était-ce le théâtre de cette dramatique histoire, que nous foulions alors sous nos pieds? Étaient-ce les neuf coups entendus et dont les ondes sonores frappaient encore à nos oreilles et versaient par là le froid à nos cœurs? Était-ce enfin tout cela combiné et confondu en moi qui m'associait à l'impression vraie de cet homme [...] ? Mais je conviens que je cessai d'être un instant du $\mathrm{XIX}^{\mathrm{e}}$ siècle, et que je crus à tout ce que m'avait dit Tainnebouy, comme il y croyait ${ }^{49}$.

Ce passage révèle aussi combien ce décor, propice au surgissement du merveilleux, est surtout un décor mental, une tournure d'esprit propre aux populations paysannes au sein desquelles se déroule l'intrigue : «C'était cette double poésie de l'inculture du sol et de l'ignorance de ceux qui la hantaient qu'on retrouvait encore, il y a quelques années, dans la sauvage et fameuse lande de Lessay", note Barbey ${ }^{50}$. Les superstitions et croyances dans les phénomènes surnaturels et les pouvoirs de certains êtres sont ainsi rappelées à de nombreuses reprises ${ }^{5 \mathrm{I}}$, comme, chez Sand, les croyances de "ces esprits avides de merveilles ${ }^{52}$ " que sont les paysans berrichons - croyances auxquelles Sand consacrera ses Légendes rustiques (1858). Dans La Petite Fadette sont par exemple évoquées celles touchant le feu follet, dont les paysans pensent «qu'il se faisait un jeu d'égarer ceux qui le regardent et de les conduire au plus creux des eaux, tout en riant à sa manière et en se moquant de leur angoisse ${ }^{53}$ ". Non seulement cette disposition d'esprit s'incarne à travers maints personnages, mais leur parlure est également restituée par le patois auquel recourent ponctuellement les deux romanciers, et qui donne à leurs récits "un goût relevé de terroir ${ }^{54}$ ».

Le merveilleux se coule ainsi tout naturellement dans le moule d'un langage et d'une culture idoines, dont le roman se fait le conservatoire. De même que les landes de Lessay sont «les lambeaux, laissés sur le sol, d'une poésie primitive et sauvage», des "[h]aillons sacrés qui disparaîtront au premier jour sous le souffle de l'industrialisme moderne", ce sont des «haillons» de pensée primitive et magique que Barbey souhaite préserver en leur offrant son roman pour réserve, lui qui comprend «les divines ignorances de l'esprit, cette poésie de l’âme qu'elle [la société] veut échanger

49. Ibid., chap. XVI, p. 74I.

50. Ibid., chap. I, p. 556.

5I. Voir par exemple chap. I, p. 557 : «Si l'on croyait les récits des charretiers qui s’y attardaient, la lande de Lessay était le théâtre des plus singulières apparitions. Dans le langage du pays, il y revenait.»

52. G. Sand, Promenades dans le Berry, G. Lubin (éd.), Bruxelles, Éditions Complexe, I992, p. 46.

53. G. Sand, La Petite Fadette, éd. citée, chap. XII, p. Io6.

54. J. Barbey d'Aurevilly, L'Ensorcelée, éd. citée, chap. I, p. 564. 
contre de malheureuses connaissances toujours incomplètes" — et l'on voit comment le merveilleux se confond ici avec une poésie qui se définit au rebours de "cet effroyable mouvement de la pensée moderne ${ }^{55}$ ». De même, il est urgent pour Sand (proche en cela de Barbey, malgré leurs divergences politiques) de préserver littérairement les traditions dont les paysans, pour quelque temps encore, sont dépositaires, traditions toujours susceptibles d'enchanter notre modernité matérialiste : «Encore un ou deux ans peut-être, et les chemins de fer passeront leur niveau sur nos vallées profondes, emportant, avec la rapidité de la foudre, nos antiques traditions et nos merveilleuses légendes", déplore-t-elle en effet dans l'"Appendice» de La Mare au Diable ${ }^{56}$.

Cet esprit favorable au merveilleux est du reste inhérent au dispositif narratif choisi par les romanciers. En effet, on se trouve dans les deux cas en présence de récits enchâssés, assumés par des narrateurs secondaires. Dans La Petite Fadette, la préface, dont on peut considérer qu'elle fait office de récit enchâssant, est énoncée par l'auteur-narrateur (dont la présence demeure ensuite en filigrane du roman); elle précise que le récit suivant est raconté par un chanvreur ${ }^{57}$, en s'achevant par ces mots : "Le chanvreur ayant bien soupé, et voyant à sa droite un grand pichet de vin blanc, à sa gauche un pot de tabac pour charger sa pipe à discrétion toute la soirée, nous raconta l'histoire suivante ${ }^{58}$.» Dans L'Ensorcelée, les deux premiers chapitres tiennent lieu de prologue, et ouvrent ensuite sur un récit qui n'est certes pas directement narré par maître Tainnebouy, le fermier cotentinais dont l'auteur-narrateur a fait la connaissance, mais qui est néanmoins présenté comme la transposition de ses paroles. Cette source énonciative est périodiquement rappelée au cours du roman, et complétée par d'autres témoignages oraux tels que celui de la comtesse de Montsurvent ${ }^{59}$; la voix du narrateur premier et celle de maître Tainnebouy tendent à se confondre, au point qu'à la fin du chapitre II, l'ensemble du récit est désigné comme "une histoire d'herbager, racontée de nuit dans une lande du Cotentin ${ }^{60}$ ", histoire dont le narrateur espère qu'elle procu-

55. Ibid., chap. I, p. 555-556.

56. Euvres complètes. $1845-1846$, t. II, B. Didier (dir.), Paris, Honoré Champion, coll. «Textes de littérature moderne et contemporaine», 20II, p. 523.

57. C'est-à-dire un broyeur de chanvre. Sur les fonctions traditionnelles du chanvreur, voir l'«Appendice» de La Mare au Diable, ibid., p. 527.

58. G. Sand, La Petite Fadette, éd. citée, p. 215.

59. Voir par exemple L'Ensorcelée, éd. citée, chap. v, p. 616 et chap. XIV, p. 723.

6o. Ibid., chap. II, p. 584 . 


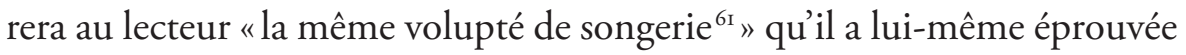
à sa première écoute.

Dans les deux cas, on assiste ainsi, d'une part, à une mise en exergue du processus énonciatif et à une démultiplication de l'instance énonciative qui recréent le dispositif du conte, caractérisé par l'oralité du récit et les échanges entre le conteur et ses auditeurs. La présence de destinataires est également présupposée dans nos récits : le chanvreur a des auditeurs (mentionnés dans la préface de La Petite Fadette) qui font figure de narrataires impliqués par l'œuvre, de même que le narrateur premier de L'Ensorcelée a été le témoin du récit de maître Tainnebouy, qu'il relaie au profit des lecteurs. D'autre part, la principale source du récit est un personnage luimême immergé dans les croyances et les superstitions locales, même s'il s'en déprend partiellement par un bon sens et une lucidité supérieurs.

\section{Polyphonie romanesque et plurivocité du sens}

C'est une dernière ruse particulièrement efficace pour crédibiliser le merveilleux et "naturaliser» son existence que cette délégation du jugement à un narrateur secondaire, qui permet à l'auteur-narrateur de ne pas se prononcer lui-même sur la réalité des phénomènes surnaturels évoqués. Celui (ou celle) de La Petite Fadette, dont la présence n'est esquissée que par la préface, ne délivre aucunement son opinion au cours du récit, assumé par le chanvreur. Ce dernier est lui-même bien moins crédule que les autres paysans dont l'opinion est citée. Il confronte par exemple aux superstitions relatives aux jumeaux l'avis du curé, qui ne voit dans leur ressemblance et leur affection fusionnelle que «les lois de la nature ${ }^{62}$ ", et l'opinion du maitre d'école, qui invoque "un phénomène de nature ${ }^{63}$ "; de même, aux légendes sur le feu follet, il oppose le point de vue rationaliste de ce maître d'école, qui n'y voit qu'un «météore» et "assure qu'on n'en doit avoir nulle crainte ${ }^{64} »$. Par ailleurs, au chapitre $\mathrm{x}$, le chanvreur se moque quelque peu de la naïveté de Landry, stupéfait que Fadette ait deviné l'endroit où il retrouverait son jumeau, en nous proposant

6I. Ibid.

62. G. Sand, La Petite Fadette, éd. citée, chap. II, p. 57.

63. Ibid., chap. v, p. 69.

64. Ibid., chap. XII, p. IO8. 
une version réaliste de cette prétendue divination ${ }^{65}$. Son scepticisme s'exprime encore au chapitre viII à propos de la mère Fadet :

Elle s'en faisait bien un peu accroire, car elle vous ôtait des maladies que vous n'aviez jamais eues, telles que le décrochement de l'estomac ou la chute de la toile du ventre, et pour ma part, je n'ai jamais ajouté foi entière à tous ces accidents-là, non plus que je n'accorde grande croyance à ce qu'on disait d'elle, qu'elle pouvait faire passer le lait d'une bonne vache dans le corps d'une mauvaise, tant vieille et mal nourrie fût-elle ${ }^{66}$.

Chez Barbey, l'entremêlement du jugement de maître Tainnebouy, qui croit manifestement au pouvoir des sorciers, et du narrateur premier rend ambiguë la position de ce dernier. Il taxe certes les croyances qui fondent le récit de "superstitions ${ }^{67}$ " au début du roman; mais son ancrage normand l'incline à les partager. Il affirme à maître Tainnebouy lorsqu'il le rencontre : "Je suis [...] du pays ${ }^{68}$ ", et c'est à un auditeur bienveillant, prédisposé à le croire, que s'adresse le fermier, lui disant : "Vous êtes du pays et v' n'êtes pas sans avoir entendu parler de certaines choses avérées parmi nous autres herbagers et fermiers... comme, par exemple, des secrets qu'ont d'aucunes personnes et qu'on appelle des sorts parmi nous ${ }^{69}$." Le narrateur de lui répondre : "Certes! oui, j'en ai entendu parler, [...] et même beaucoup dans mon enfance. J'ai été bercé avec ces histoires... ${ }^{70}$ ", soulignant que l'intérêt du récit le ramène aux charmes d'une enfance entourée d'affection (la métaphore du verbe «bercer» associe ces histoires à la tendresse prodiguée par la mère ou la nourrice). Un peu plus loin, enfin, il affirme sans ambages son goût pour les traditions merveilleuses : "J'ai toujours été grand amateur et dégustateur de légendes et de superstitions populaires, lesquelles cachent un sens plus profond qu'on ne croit, inaperçu par les esprits superficiels ${ }^{71} \ldots »$

Il refuse ainsi de révoquer clairement en doute ces «légendes» et ménage les différentes interprétations possibles, soit l'hypothèse d'un ensorcellement (dont l'origine est elle-même en débat), soit une explication de type psychologique :

65. Voir ibid., p. 94 : «L'idée ne lui vint pas qu'il avait déjà demandé son frère à plusieurs personnes en venant à la Joncière, et que quelqu'un avait pu en parler devant la petite Fadette; ou bien, que cette petite pouvait avoir écouté la fin de son discours avec la grand-mère, en se cachant comme elle faisait souvent pour connaître tout ce qui pouvait contenter sa curiosité. »

66. Ibid., p. 83 .

67. J. Barbey d'Aurevilly, L'Ensorcelée, éd. citée, chap. I, p. 556.

68. Ibid., chap. II, p. 568.

69. Ibid., chap. II, p. 574 .

70. Ibid., chap. II, p. 574 .

7I. Ibid., chap. II, p. 583 . 
[...] s'il y avait dans l'histoire de l'herbager [maître Tainnebouy] ce qu'on nomme communément du merveilleux (comme si l'envers, le dessous de toutes les choses humaines n'était pas du merveilleux tout aussi inexplicable que ce qu'on nie, faute de l'expliquer!), il y avait en même temps de ces événements produits par le choc des passions ou l'invétération des sentiments, qui donnent à un récit, quel qu'il soit, l'intérêt poignant et immortel de ce phénix des radoteurs dont les redites sont toujours nouvelles, et qui s'appelle le cœur de l'homme ${ }^{72}$.

Ce commentaire du narrateur établit une concomitance, voire une équivalence entre les deux plans, la parenthèse soulignant que les ressorts des actions humaines ne sont parfois pas moins obscurs que les phénomènes tenus pour surnaturels. C'est donc autant par intérêt personnel que pour se mettre en conformité avec les codes herméneutiques de son temps et ne pas froisser les habitudes de pensée du lecteur, que le narrateur se penche sur les mobiles psychologiques de Jeanne :

Ferai-je bien comprendre ce caractère? Si on ne le comprenait pas, ce récit serait incroyable. On serait alors obligé d'en revenir aux idées de maître Tainnebouy, et ces idées ne sont plus dans la donnée de notre temps. Pour l'observateur qui s'abîme dans le mystère de la passion humaine et de ses sources, elles n'étaient pas plus absurdes qu'autre chose, mais le scepticisme d'un siècle comme le nôtre les repousserait ${ }^{73}$.

Parallèlement au fil surnaturel, un autre fil permet ainsi au lecteur de reconstituer en termes psychologiques le processus passionnel qui finit par aliéner Jeanne Le Hardouey — processus si mystérieux, malgré les explications avancées, qu'il faut parler d'une intériorisation, mais non d'une destruction du merveilleux. L' « espèce de vertige» et la «fascination pleine d'angoisse » qu' elle éprouve à l'église de Blanchelande devant «la terrible tête [de l'abbé] encadrée dans [son] capuchon noire» sont d'abord signalés ${ }^{74}$; puis la prédiction du berger précipite la cristallisation passionnelle de la sensation inconnue qu'a éveillée chez Jeanne l'apparition de l'abbé : "Le mot du berger [...], venant après l'impression qu'elle avait reçue pendant les vêpres, la redoublait et la faisait fermenter en elle ${ }^{75}$." Amplifié par les nombreuses rumeurs entourant l'abbé, qu'elle écoute "avec une horreur passionnée ${ }^{76}$ ", son sentiment se mêle ensuite d'admira-

72. Ibid.

73. Ibid., chap. vIII, p. 647.

74. Ibid., chap. IV, p. 603.

75. Ibid., chap. vi, p. 62I.

76. Ibid., chap. vI, p. 628. 
tion pour l'ancien Chouan, tout en renvoyant Jeanne, née de Feuardent, à la honte de sa mésalliance ${ }^{77}$.

Alors qu' elle le rencontre seul chez Clotilde Mauduit (qui, dans sa jeunesse, a fréquenté Jéhoël de la Croix-Jugan et le père de Jeanne au château du Haut-Mesnil), l'émotion de Jeanne atteint une telle intensité qu'une vive rougeur envahit son visage et ne le quittera plus. Une caractérisation clinique du phénomène est alors opérée, à travers un abondant lexique physiologique : "Sur sa pâleur sortaient de partout des taches rouges, un semis de plaques ardentes [...]. À chaque mot, à chaque geste de l'abbé, apparaissaient ces taches effrayantes. Il y en avait sur le front, aux joues. Plusieurs se montraient déjà sur le cou et sur la poitrine... ${ }^{78}$ »; on trouve encore un tel vocabulaire dans les mentions d'une "couperose ardente de son sang soulevé79", ou de "ce malheureux visage, affreux comme une apoplexie ${ }^{80}$ ".

Ce phénomène, qu'on qualifierait de psychosomatique ${ }^{81}$, est par ailleurs expliqué par l'ascendance de Jeanne :

Louisine avais transmis à sa fille la force d'âme qui respirait en elle comme un souffle de divinité; mais, pour le malheur de Jeanne-Madeleine, il s'y mêlait le sang des Feuardent, d'une race vieillie, ardente autrefois comme son nom, et ce sang devait produire en elle quelque inextinguible incendie, pour peu qu'il fût agité par cette vieille sorcière de Destinée qui remue si souvent nos passions dans nos veines endormies, avec un tison enflammés

Quoiqu'en ait Barbey, qui haïssait Zola et son «impudent matérialisme ${ }^{8_{3}}$ », un romancier naturaliste n'eût pas désavoué un tel passage, même si l'idée de prédestination induite par l'onomastique (le patronyme Feuardent semble sceller le sort de l'héroïne) et le thème de la destinée sont d'un autre registre! Ce nonobstant, la présence de l'hérédité, la "pathologisa-

77. Voir ibid., chap. vII, p. 630 : "L'horreur y était toujours, mais, chez cette femme d'action et de race, qui ne s'était jamais consolée d'avoir humilié la sienne dans une mésalliance, l'admiration pour ce moine décloîtré par la guerre civile, qui ne s'était souvenu que d'une chose, au prix du salut de son âme, c'est qu'il était gentilhomme, oui, l'admiration l'emportait alors sur l'horreur...»

78. Ibid., chap. viII, p. 645 .

79 Ibid., chap. viII, p. 65I.

80 Ibid., chap. x, p. 670.

8I. Il est question des «révélations d'affreux troubles dans ce malheureux cœur volcanisé» (ibid., chap. viII, p. 65I), ou encore de "ce masque rouge de sang extravasé, que les révoltes d'un cœur trop concentré avaient moulé sur son visage» (ibid., chap. IX, p. 658).

82. Ibid., chap. v, p. 615.

83. J. Barbey d'Aurevilly, Les CEuvres et les Hommes ( $3^{e}$ série) - XVIII. Le Roman contemporain, Paris, Lemerre, 1902, p. 223. 
tion " du merveilleux ${ }^{84}$ évoquée ci-dessus et son lien avec la folie ${ }^{85}$ ancrent bien L'Ensorcelée dans un courant d'idées et un imaginaire qui irriguent la seconde moitié du Xix ${ }^{e}$ siècle ${ }^{86}$, et qu'on retrouve par exemple dans les contes fantastiques de Maupassant.

\section{Deux visions du monde contrastées}

Les explications rationnelles de type psychologique ou psychopathologique sont néanmoins tenues par Barbey pour insuffisantes :

La science moderne, qui a pris connaissance de ces faits et qui les explique ou croit les expliquer [le romancier vient de mentionner un autre cas d'ensorcellement d'une jeune fille noble par un prêtre, au XvII ${ }^{e}$ siècle], ne trouvera jamais le secret de l'influence d'un être humain sur un autre être humain dans des proportions aussi colossales. En vain prononce-t-on le mot d'amour. On veut éclairer un abîme par un second abîme qu'on creuse dans le fond du premier ${ }^{87}$.

En revanche, une dernière hypothèse explicative semble privilégiée par le narrateur de L'Ensorcelée, et s'impose d'autant plus évidemment au lecteur s'il relie ce roman aux autres textes, fictionnels ou critiques, de Barbey d'Aurevilly, qui, du moins à partir des années I840, n’a pas cessé de proclamer un catholicisme intransigeant. Cette dernière hypothèse est celle d'une influence efficiente du Diable sur la vie des hommes, attestée par l'Église, comme le souligne cette profession de foi du narrateur, qui clôt le récit enchâssant du roman :

Quoique je ne partageasse pas toutes ses idées [de maitre Tainnebouy] [...], cependant j'étais bien loin de les repousser, car j'ai toujours cru, d'instinct autant que de réflexion, aux deux choses sur lesquelles repose en définitive la magie, je veux dire : à la tradition de certains secrets [...] et à l'intervention des puissances occultes

84. Chez Sand, c'est la passion exclusive de Sylvinet pour son jumeau qui a des traits pathologiques. On lit au chapitre XXXI : «Le pauvre Sylvinet, dont la fantaisie était de posséder à lui tout seul l'amitié de ceux qu'il aimait, tomba dans un ennui mortel, dans une langueur singulière, et son esprit se rembrunit si fort qu'on ne savait par où le prendre pour le contenter. Il ne riait plus jamais; il ne prenait goût à rien, il ne pouvait plus guère travailler, tant il se consumait et s'affaiblissait. Enfin on craignit pour sa vie, car la fièvre ne le quittait presque plus [...]. Il souhaitait la mort..." (La Petite Fadette, éd. citée, p. 178-179.)

85. Voir par exemple L'Ensorcelée, éd. citée, chap. x, p. 67I : Jeanne se précipite hors de la chaumière de la Clotte "comme une folle qui s'échapperait de l'hôpital".

86. Le fantastique a partie liée avec l'obsession, l'hallucination ou la folie, dans un contexte où les progrès de la médecine stimulent l'intérêt pour les maladies mentales et leurs conséquences. Voir notamment sur ces questions G. Ponnau, La Folie dans la littérature fantastique, Paris, PUF, coll. «Écriture», I997.

87. J. Barbey d'Aurevilly, L'Ensorcelée, éd. citée, chap. IX, p. 65I. 
et mauvaises dans les luttes de l'humanité. J'ai pour moi dans cette opinion l'histoire de tous les temps et de tous les lieux, à tous les degrés de la civilisation chez les peuples, et, ce que j'estime infiniment plus que toutes les histoires, l'irréfragable attestation de l'Église romaine, qui a condamné, en vingt endroits des actes de ses Conciles, la magie, la sorcellerie, les charmes, non comme choses vaines et pernicieusement fausses, mais comme choses RÉELLEs, et que ses dogmes expliquaient très bien. Quant à l'intervention de puissances mauvaises dans les affaires de l'humanité, j'ai encore pour moi le témoignage de l'Église... ${ }^{88}$.

Le responsable de l'ensorcellement de Jeanne serait donc le Diable luimême, dont les bergers seraient les affidés et dont l'abbé de la Croix-Juan est présenté comme le servant, voire l'incarnation - ce qui expliquerait "l'ascendant surnaturel de cet homme ${ }^{89}$ ": «Il a plutôt l'air d'un diable que d'un prêtre ${ }^{\circ} "$ ", juge d'emblée Thomas Le Hardouey. Quand bien même on ramènerait le sort de Jeanne à des causes psychiques, ce sont des péchés qui font d'elle la proie du Démon ${ }^{91}$, la concupiscence, le désir charnel, mais avant tout l'Orgueil, orgueil nobiliaire ${ }^{92}$ dont Jéhoël de la Croix-Jugan est lui-même dévoré : "Ce singulier prêtre [...] avait le secret de consoler par l'orgueil les âmes ulcérées, comme s'il avait été un ministre de Lucifer au lieu d'être l'humble prêtre de Jésus-Christ ${ }^{93}$ ", affirme le narrateur. Tout l'univers de L'Ensorcelée (comme des autres œuvres de Barbey) est ainsi sous-tendu par la postulation métaphysique du Mal et de l'existence d'un surnaturel essentiellement diabolique.

Au contraire, dans La Petite Fadette, le merveilleux est en dernière instance un merveilleux naturel (si cet oxymore a un sens) qui évacue l'hypothèse du surnaturel ${ }^{94}$. Les faits tenus pour prodigieux sont des phénomènes

88. Ibid., chap. II, p. 583-584.

89. Ibid., chap. viII, p. 644 .

90. Ibid., chap. vi, p. 625 .

9I. "[...] oui, je crois que les vices qu'on a eus vous ensorcellent», déclare ainsi la Clotte (ibid., chap. vII, p. 639).

92. La rougeur de Jeanne est le signe obvie de sa honte devant l'ancien Chouan qui n'a jamais renié sa cause : «L'idée de son mariage, de sa chute volontaire dans les bras d'un paysan, lui fondait le front dans le feu de la honte", lit-on au chapitre x (ibid., p. 646); et après la mort de Jeanne, le narrateur évoque "cette fille noble qui avait emprisonné dans un corset de bure une âme patricienne longtemps contenue, longtemps surmontée, et qui tout à coup, éclatant à l'approche d'une âme de sa race, avait tué son bonheur et brisé sa vie!» (ibid., chap. xIII, p. 699).

93. Ibid., chap. viII, p. 648.

94. Selon Sylvie Veys, cette tendance est notable jusque dans les Contes d'une grand-mère: «Peu présentes en fin de compte, souvent métaphoriques, [les fées] semblent disparaître progressivement, cédant la place à la volonté humaine et aux forces de la nature. [...] Le merveilleux n'est pas tant leur apanage que celui de la nature généreuse et éternelle.» «Du populaire au littéraire : constantes et variations des Contes d'une grand-mère", dans George Sand. Pratiques et imaginaires de l'écriture, ouvr. cité, p. I4O-I4I. 
de nature. C'est le cas des «bessons» (ou jumeaux), «si pareils» qu' «[on] dirait deux petits perdreaux sortant de l'œuf $95^{\prime}$ ", que "tous les gens des alentours [...] s'arrêtaient pour les regarder, pour s'émerveiller de leur retirance [ressemblance] ${ }^{96} "$, et qui font l'objet de diverses superstitions : "J'ai ouï-dire que les bessons prenaient tant d'amitié l'un pour l'autre, que quand ils se quittaient ils ne pouvaient plus vivre, et qu'un des deux, tout au moins, se laissait consumer par le chagrin, jusqu'à en mourir ${ }^{97}$ ", déclare ainsi le père Barbeau. Quant aux prédictions qui scandent le roman - celle de la Sagette, qui consiste en la mise en garde rapportée ci-dessus par le père Barbeau, ou celle de la Baigneuse au sujet de Sylvinet ${ }^{98}$ —, et qui se trouvent vérifiées par l'intrigue, elles sont mises sur le compte d'une sagesse supérieure des femmes qui les profèrent : le nom seul de la Sagette est éloquent, et la prédiction de la Baigneuse, rappelée à la fin du roman, est attribuée au "grand jugement [qu'elle avait] sur toutes les maladies du corps et de l'esprit ${ }^{99} »$.

De la même façon, c'est aux connaissances ${ }^{100}$ et au jugement de la mère Fadet que le chanvreur ramène sa prétendue sorcellerie. Il affirme :

Comme, dans la campagne, on n'est jamais savant sans être quelque peu sorcier, beaucoup pensaient que la mère Fadet en savait encore plus long qu' elle ne voulait le dire [...]; enfin, de ce qu'elle avait beaucoup d'esprit et de raisonnement pour vous aider à sortir de peine dans beaucoup de choses possibles, on inférait qu'elle pouvait en faire d'autres qui ne le sont pas $^{\text {Ior }}$.

C'est de même le bon sens et l'esprit supérieur de Fanchon Fadet — son "esprit du diable ${ }^{\mathrm{IO}} » !$ - qui l'apparentent aux yeux des ignorants à une sorcière ou à une fée; le narrateur finit par convenir qu' elle possède un "don de nature» qui, joint aux leçons de sa grand-mère, lui permettent de "découvr[ir] et devin[er] comme qui invente, les vertus que le bon Dieu a mises dans certaines herbes et dans certaines manières de les employer.

95. G. Sand, La Petite Fadette, éd. citée, chap. I, p. 53.

96. Ibid., chap. II, p. 56.

97. Ibid., chap. I, p. 53.

98. Voir ibid., chap. xxxI, p. 179 : «Sylvinet, le jour où il aimera une femme, l'aimera encore plus follement qu'il n'aime son frère. Je vous prédis cela.»

99. G. Sand, La Petite Fadette, éd. citée, chap. xxxI, p. I79.

Ioo. Selon Simone Vierne, c'est « une forme de connaissance purement paysanne, une connaissance primitive» qui intéresse avant tout George Sand, connaissance qui à ses yeux «n'est pas moins intéressante que la science matérialiste moderne». "Fées traditionnelles et femmes modernes chez George Sand", dans Images de la magie. Fées, enchanteurs et merveilleux dans l'imaginaire du $X I X^{e}$ siècle, ouvr. cité, p. 52.

IOI. G. Sand, La Petite Fadette, éd. citée, chap. viII, p. 84.

IO2. Ibid., chap. XxxIII, p. I86. 
Elle n'était point sorcière pour cela [...]; mais elle avait l'esprit qui observe, qui fait des comparaisons, des remarques, des essais, et cela c'est un don de nature $[\ldots]^{103}$ ".

La petite Fadette elle-même, rejetant les accusations de sorcellerie et au rebours d'un imaginaire diabolique, situe son savoir et l'usage qu'elle en fait dans le cadre d'une vision chrétienne et d'une charité étendue au monde naturel, animal et végétal. Elle expose son credo à Landry au chapitre XVIII :

Moi je sais, sans être sorcière, à quoi sont bonnes les moindres herbes que tu écrases sous tes pieds; et quand je sais leur usage, je les regarde et ne méprise ni leur odeur ni leur figure. Je te dis cela, Landry, pour t'enseigner tout à l'heure une autre chose qui se rapporte aux âmes chrétiennes aussi bien qu'aux fleurs des jardins et aux ronces des carrières; c'est que l'on méprise trop souvent ce qui ne paraît ni beau ni bon, et que par là on se prive de ce qui est secourable et salutaire. [...] on dit que j'aime les mauvaises bêtes et que je suis sorcière, parce que je n'aime pas à faire souffrir une grenouille, à arracher les pattes d'une guêpe et à clouer une chauve-souris vivante contre un arbre ${ }^{\mathrm{IO} 4}$.

«Elle a un esprit merveilleux, et un cour comme le bon Dieu n'en fait pas souvent ${ }^{105}$ ", conclura Landry quand il la connaîtra mieux.

Quant au phénomène le plus étonnant du roman, la guérison de Sylvinet par de simples impositions des mains de Fanchon, celle-ci l'attribue à sa foi : «Elle s'imaginait que l'amitié et la volonté d'une personne en bonne santé, et l'attouchement d'une main pure et bien vivante, peuvent écarter le mal, quand cette personne est douée d'un certain esprit et d'une grande confiance dans la bonté de Dieu ${ }^{106}$.» Elle met ainsi "plus de religion que de diablerie dans ses charmes ${ }^{107}$ ", comme le souligne le chanvreur, qui suggère quant à lui un phénomène de suggestion mentale ${ }^{108}$ en invoquant "sa grande volonté ${ }^{109}$ ". "Miracle ou non", conclut philosophiquement le père Barbeau, "cette fille-là a un grand esprit, et je crois bien que ça doit porter bonheur de l'avoir dans une famille ${ }^{\mathrm{IIO}}$ "!

\footnotetext{
I03. Ibid., chap. XXVI, p. I59.

I04. Ibid., chap. XVIII, p. I28-I30.

I05. Ibid., chap. XXI, p. I43.

Io6. Ibid., chap. xxxv, p. 193.

I07. Ibid., chap. xxxv, p. 194.

I08. On pourrait également penser à une forme de magnétisme.

I09. G. Sand, La Petite Fadette, éd. citée, chap. XxxvII, p. 214.

IIo. Ibid., chap. XL, p. 207.
} 
Non seulement aucune créance, dans l'univers sandien, n'est accordée au surnaturel diabolique ${ }^{\mathrm{III}}$, mais, contrairement encore à ce qu'on note chez Barbey - pour qui tout ne peut aller que de mal en pis - l'univers de La Petite Fadette est foncièrement optimiste. On peut dire de ce roman qu'il décrit "sous une forme imaginaire et symbolique les étapes essentielles de la croissance et de l'accession à une vie indépendante ${ }^{\mathrm{II} 2}$ ", et sa structure révèle cette "orientation éthique» et cette "morale naïve», profondément satisfaisante, qu'André Jolles considère comme des caractéristiques du conte merveilleux ${ }^{\mathrm{Ir}}$. Ainsi, on assiste dans le roman à une double initiation parfaitement réussie. D'une part, Fanchon est éveillée par Landry à l'amour et "le follet maléfique, et masculin, devient une sage jeune fille, mais aussi une guérisseuse, qui rompt le charme unissant les deux bessons, et les transforme à son tour ${ }^{\mathrm{II} 4}$ ». D'autre part, en effet, Landry accède au bonheur, à la sagesse et à l'aisance matérielle grâce à Fanchon, dont l'amitié puis l'amour viennent couronner la réussite par le jeune homme de toute une série d'épreuves.

Comme dans un conte, et avant d'être récompensé de sa patience et de sa soumission aux souhaits de la jeune femme, Landry est récompensé d'avoir tenu parole à la petite fille. Celle-ci, à la manière d'une fée déguisée, a exigé de lui un gage lorsqu'elle l'a tiré, par deux fois, d'affaire. D’abord en l'aidant à retrouver son frère, au chapitre IX; après lui avoir révélé l'endroit où Landry le retrouverait, elle le quitte en lui enjoignant : «N'oublie point que je ne te réclamerai rien jusqu'au jour où je me serai décidée à t'aller trouver pour te requérir d'une chose qui sera à mon commandement et que tu feras sans retard ni regret ${ }^{\mathrm{II}}$." Puis en l'aidant à traverser le gué, le sauvant de la noyade, au chapitre XII, où elle intervient telle un lutin, en chantonnant comme pour conjurer le feu follet ${ }^{116}$. Après avoir reproché à

\footnotetext{
III. Fanchon réfute toute action du Diable sur les hommes, dans une profession de foi qu'elle énonce devant Landry : "S'il existe [le diable], je suis bien assurée qu'il n'a aucun pouvoir pour venir sur la terre nous abuser et nous demander notre âme pour la retirer du bon Dieu. [...] puisque la terre est au Bon Dieu, il n'y a que le bon Dieu qui puisse gouverner les choses et les hommes qui s'y trouvent.» (Ibid., xxv, p. I57.)

II2. C'est ainsi que Bruno Bettelheim définit le conte de fées (Psychanalyse du conte de fées (1976), traduit de l'anglais par T. Carlier, Paris, Robert Laffont, coll. «Le Livre de Poche», 1976, p. 132). II3. «Il y a dans le conte une forme où l'événement et le cours des choses suivent un ordre tel qu'ils répondent entièrement aux exigences de notre morale naïve et qu'ils sont donc "bons" et "justes" selon notre jugement sentimental absolu», affirme André Jolles. ("Le conte», dans Formes simples [1930], traduit de l'allemand par A. M. Buguet, Paris, Éditions du Seuil, coll. «Poétique», 1972, p. 190.)

II4. S. Vierne, «Fées traditionnelles et femmes modernes chez George Sand», art. cité, p. 57.

II5. G. Sand, La Petite Fadette, éd. citée, p. 9I.

II6. Ibid., p. 107.
} 
Landry de ne pas lui avoir adressé un seul mot de remerciement pour son précédent bienfait, elle lui intime : «À présent je vous réclame ce que vous m'avez promis, qui est d'obéir à mon commandement, le jour où vous en serez requis ${ }^{\mathrm{II}}$. " Or la promesse de Landry — qu'à la Saint-Andoche, il lui fasse "danser trois bourrées après la messe, deux bourrées après vêpres, et encore deux bourrées après l'Angélus, ce qui fera sept ${ }^{\mathrm{II} 8}$ » - sera remplie et même au-delà, puisque le jeune homme la défendra en sus contre les brutalités de galopins et la sauvera de l'humiliation en la menant fièrement danser avec lui : "À présent, c'est assez, Landry, je suis contente de toi, et je te rends ta parole ${ }^{\mathrm{II} 9}$ ", lui dit alors Fanchon. Ainsi, de même que la petite Fadette est une "Cendrillon qui a une marraine Fanchette trop simple pour l'aider et qui se tire elle-même de la misère ${ }^{120}$ ", Landry doit d'abord sa récompense à la force et à la bonté d'âme qu'il a démontrées en diverses occasions ${ }^{121}$.

Quant au dénouement du roman, on l'a déjà suggéré, c'est bien à la manière d'un conte qu'il est un happy end des plus réconfortants : les bons sont récompensés et les méchants... Mais y a-t-il vraiment des méchants dans La Petite Fadette? La Madelon elle-même, certes vaniteuse, coquette et inconstante, s'avère une adjuvante pour les deux héros puisqu'en éconduisant Landry, elle contribue à le pousser dans les bras de Fanchon ${ }^{122}$. L'univers sandien a bien dans ce roman des teintes idylliques; l'auteure le reconnait dans les paratextes de l'œuvre, où elle avoue avoir voulu peindre " un certain idéal de la vie champêtre [...] naïf et enfantin ${ }^{123}$ ", de façon à procurer au lecteur un "soulagement passager ${ }^{124}$ ». En effet, juge-t-elle, "mieux vaut une douce chanson, un son de pipeau rustique, un conte pour endormir les petits enfants sans frayeur et sans souffrance, que le spectacle des maux réels renforcés et rembrunis par les couleurs de la fic-

II7. Ibid., chap. XIII, p. II2.

II8. Ibid.

I19. Ibid., chap. XvI, p. I22.

I20. A. Mansau, "Présentation», dans La Petite Fadette, éd. citée, p. I9.

I2I. Sylvie Veys montre que même dans ses Contes d’une grand-mère, "George Sand veut leur faire comprendre [à ses lecteurs] que, conte merveilleux ou pas, le héros doit travailler, lutter, assumer ses choix et ses responsabilités, sans s'en dédouaner sur un auxiliaire magique». "Du populaire au littéraire : constantes et variations des Contes d'une grand-mère», art. cité, p. 137.

I22. Voir La Petite Fadette, éd. citée, chap. Xxi. Béatrice Didier note que de même, dans les Contes d'une grand-mère, "[l]es opposants ne sont guère cruels". "Le génie narratif des Contes", Littérature, $\mathrm{n}^{\circ} \mathrm{I} 34$ (George Sand: "Le génie narratif»), 2004, p. II6.

I23. G. Sand, La Petite Fadette, éd. citée, Préface de l'édition originale, p. 2 I4.

I24. Ibid., «Notice», p. 49. 
$\operatorname{tion}^{125}$ ». «Nous croyons que [...] le roman d'aujourd'hui devrait remplacer la parabole et l'apologue des temps naïfs ${ }^{\mathrm{I2}}{ }^{\prime}$, avait-elle déjà affirmé dans le premier chapitre de La Mare au Diable.

Au terme de ce parcours, on comprend mieux le mépris que professa Barbey pour Sand, tenant à des raisons plus idéologiques encore qu'esthétiques, Sand étant "un des visages de cet Infâme moderne que Barbey a pris pour tâche d'écraser ${ }^{127}$ ». Éloignée du catholicisme, Sand croit en un merveilleux qui se confond avec la nature; celui-ci se coule harmonieusement dans le cadre champêtre de ses romans, qui sont en outre orientés par la logique même, optimiste, du conte — du fait de ce que Barbey taxerait d'idéalisme de la part de l'auteure. Le pessimiste auteur des Diaboliques croit au contraire en la présence agissante du Diable et de ses démons dans le cœur des hommes, que leurs passions livrent au Malin : pas d'initiation réussie chez lui, mais l'inéluctable Chute des proies de Lucifer. Parmi les héros de L'Ensorcelée $e^{128}$, pourrait-on dire, il n'y a que des Méchants - à l'exception de Clotilde Mauduit, sauvée par sa conversion in extremis? - ou plutôt de misérables victimes de Satan, dont la plus déplorable est sans doute l'abbé de la Croix-Jugan, condamné à redire pour l'Éternité sa messe inachevée ${ }^{\mathrm{I} 2}$. La communication des deux sphères, humaine et surnaturelle, et l'indécision maintenue entre ces deux plans sont aussi les sources d'un climat qui tient davantage au fantastique qu'au merveilleux : toute l'action de L'Ensorcelée se joue à la frontière de ces deux mondes; tandis que dans La Petite Fadette - comme dans La Mare au Diable - l'ombre des maléfices se dissipe prestement aux lumières naturelles de l'esprit et du cœur humains.

I25. Ibid., p. 48.

I26. G. Sand, La Mare au Diable, chap. I, dans Euvres complètes. I845-1846, éd. citée, t. II, p. 42I. I27. Ph. Berthier, "L'inquisiteur et la dépravatrice : Barbey d'Aurevilly et George Sand (I850-1889) », Revue d'Histoire littéraire de la France, $79^{\mathrm{e}}$ année, ${ }^{\circ}$ I, janvier-février 1979, p. 50.

I28. D'autres de ses romans accueillent des êtres angéliques, par exemple Calixte dans Un prêtre marié.

I29. L'horrible vision de ce damné est rapportée par le forgeron Pierre Cloud, dans un récit que relaie maître Tainnebouy au narrateur premier. Voir L'Ensorcelée, éd. citée, chap. XVI, p. 737-74I. 
